\title{
SOME MORE MATHIEU GROUP COVERINGS IN CHARACTERISTIC TWO
}

\author{
SHREERAM S. ABHYANKAR AND IKKWON YIE
}

(Communicated by Ronald M. Solomon)

\begin{abstract}
Explicit equations are given for unramified coverings of the affine line in characteristic two with Mathieu groups of degrees 23 and 24 as Galois groups.
\end{abstract}

\section{INTRODUCTION}

Let $k$ be a field of characteristic $p \neq 0$, and consider the polynomial $\bar{F}_{23,20,1,1}=Y^{23}+X Y^{3}+1$ of degree 23 in $Y$ with coefficients in $k[X]$. Inspired by Serre's linearization trick (cf. [6] as reported in Section 1 of [5]), in the case of $p=2$, in (1.5) of [4] a linearization lemma was proved for this polynomial and, together with the transitivity lemma (1.3) of [4], it showed that the said polynomial gives an unramified covering of the affine line $L_{k}$ (in characteristic two) having $M_{23}$ (= the Mathieu group of degree 23) as Galois group. In the present paper, by modifying this procedure, we shall prove the following:

First Mathieu Group Theorem (1.1). If $p=2$ then, for any $\alpha \in k$, the Galois group $\operatorname{Gal}\left(Y^{24}+\alpha Y^{4}+Y+X, k(X)\right)$ equals the Mathieu group $M_{24}$ of degree 24.

From (1.1) it follows that, for $p=2$, the equation $Y^{24}+\alpha Y^{4}+Y+X=0$ gives an unramified covering of the affine line $L_{k}$ with Galois group $M_{24}$. It may be noted that this covering is a special case of the family of unramified coverings given in Proposition 2 of the 1957 paper [1]. Moreover, the subcase $\alpha=0$ is part of the tilde family on pp. 74 and 103-108 of [2] and in (9.5) of [3] it was called a border value case giving interesting Galois group. In the subcase $\alpha=0$, recently McKay and Conway have independently shown the Galois group to be $M_{24}$.

By "throwing away" a root of the above equation [see (2.1)], by (1.1) we get the following:

Received by the editors March 30,1993.

1991 Mathematics Subject Classification. Primary 12F10, 14H30, 20D06, 20 E22.

The first author's work was partly supported by NSF grant DMS 91-01424 and NSA grant MDA 904-92-H-3035. The second author's work was partly supported by PRF grant 690-1395-1920. 
Second Mathieu Group Theorem (1.2). If $p=2$ and $\alpha$ is any element of $k$ then upon letting $\Phi=Y^{-1}\left[(Y+X)^{24}-X^{24}\right]+\alpha Y^{-1}\left[(Y+X)^{4}-X^{4}\right]+1=$ $Y^{23}+X^{8} Y^{15}+X^{16} Y^{7}+\alpha Y^{3}+1$, the equation $\Phi=0$ gives an unramified covering of the affine line $L_{k}$ with $\operatorname{Gal}(\Phi, k(X))=M_{23}$.

To explain the linearization trick, let us recall that an additive polynomial $\boldsymbol{\theta}$ over a domain $D$ of characteristic $p$ is a polynomial of the form $\boldsymbol{\theta}=$ $Y^{p^{M}}+\sum_{i=0}^{M-1} a_{i} Y^{p^{i}}$ where $a_{i} \in D$ with $a_{0} \neq 0$. Clearly the $Y$-derivative of $\theta$ equals the nonzero constant $a_{0}$ and hence, for any overfield $\Lambda$ of $D$, the Galois group $\operatorname{Gal}(\boldsymbol{\theta}, \Lambda)$ is defined. Moreover, the roots of $\boldsymbol{\theta}$ obviously form an elementary abelian group of order $p^{M}$ and hence, as a permutation $\operatorname{group}, \operatorname{Gal}(\Theta, \Lambda)$ is a subgroup of $\operatorname{GL}(M, p)$. Now let there be given a monic polynomial $\Gamma$ of degree $N>0$ in $Y$ with coefficients in $D$. Assume that the $Y$-discriminant of $\Gamma$ is nonzero so that we can talk about the Galois group $\operatorname{Gal}(\Gamma, \Lambda)$. We shall say that $\Gamma$ linearizes over $D$ at $M$ if there exists an additive polynomial $\Theta$ over $D$ of degree $p^{M}$ such that $\Gamma^{*}=\theta$ for some $\Gamma^{*} \in D[Y]$. If this is so, then $\operatorname{Gal}(\Gamma, \Lambda)$ is a homomorphic image of $\operatorname{Gal}(\boldsymbol{\theta}, \Lambda)$ and hence the order $|\operatorname{Gal}(\Gamma, \Lambda)|$ of $\operatorname{Gal}(\Gamma, \Lambda)$ divides the order $|\operatorname{GL}(M, p)|$ of $\operatorname{GL}(M, p)$. It is easily seen that $\Gamma$ always linearizes over $\Lambda$ at $M=N$. But if it linearizes at a significantly smaller value of $M$ then we can obtain reasonable bounds for the prime power factors of $|\mathrm{Gal}(\Gamma, \Lambda)|$.

In (1.5) of [4] it was shown that, for $p=2$, the polynomial $\bar{F}_{23,20,1,1}=$ $Y^{23}+X Y+1$ linearizes over $k[X]$ at 11 . In the Linearization Lemma (5.1) of Section 5, by slightly modifying the proof of (1.5) of [4], we shall show that, for $p=2$ and for any element $T$ in an overfield of $k[X]$ (for instance, $T$ could be transcendental over $k[X])$, the polynomial $F^{*}=Y \bar{F}_{23,20,1,1}+T$ linearizes over $k[X, T]$ at 12 . By taking $(\alpha, X)$ for $(X, T)$ in $F^{*}$, it follows that $\left|\mathrm{Gal}\left(Y^{24}+\alpha Y^{4}+Y+X, k(X)\right)\right|$ divides $|\mathrm{GL}(12,2)|$.

For $p=2$, in (1.3) of [4] it was shown that $\mathrm{Gal}\left(Y^{23}+X Y^{3}+1, k(X)\right)$ is doubly transitive and, as said above, this together with the fact that $Y^{23}+X Y^{3}+$ 1 linearizes at 11 shows that $\operatorname{Gal}\left(Y^{23}+X Y^{3}+1, k(X)\right)=M_{23}$. This time, in the Transitivity Lemma (4.1) of Section 4, we shall show that for certain monic polynomials $F$ of degree $n=m q$ in $Y$ with coefficients in $k[X]$ where $q$ is the highest power of $p$ which divides $n$ and where $p$ need not be 2 , the Galois group $\operatorname{Gal}(F, k(X))$ is doubly transitive and its order is divisible by $n(n-1)(q-1)$. The proof of the Transitivity Lemma (4.1) will be based on some auxilliary lemmas which we shall prove in Section 2 and an irreducibility lemma which we shall prove in Section 3. Thus the Linearization Lemma (5.1) shows that $\operatorname{Gal}\left(Y^{24}+\alpha Y^{4}+Y+1, k(X)\right)$ is not too big and the Transitivity Lemma (4.1) shows that it is not too small. In Section 6, Theorem (1.1) will be deduced from these two facts.

\section{AuXilliary LEMMAS}

Let $F=F(Y)=Y^{n}+B_{n_{1}} Y^{n_{1}}+B_{n_{2}} Y^{n_{2}}+\cdots+B_{n_{h}} Y^{n_{h}}+X$ where $h$ and $n>n_{1}>n_{2}>\cdots>n_{h}=1$ are positive integers, and $0 \neq B_{n_{i}} \in k$ for $1 \leq i \leq h$. Assume that $n$ is divisible by $p$ and let $m$ and $q$ be the unique positive integers with $n=m q$ such that $m$ is nondivisible by $p$ and $q$ is a power of $p$. For $1 \leq i \leq h-1$ assume that $n_{i}$ is divisible by $p$ and let $m_{i}$ and $q_{i}$ be the unique positive integers with $n_{i}=m_{i} q_{i}$ such that $m_{i}$ is nondivisible 
by $p$ and $q_{i}$ is a power of $p$. [Note that the $Y$-derivative of $F$ equals the nonzero element $B_{n_{h}}$ of $k$ and hence the Galois group $\operatorname{Gal}(F, k(X))$ makes sense and the equation $F=0$ gives an unramified covering of the affine line $\left.L_{k} \cdot\right]$

In the Transitivity Lemma (4.1) of Section 4 we shall show that if certain conditions are satisfied then $\operatorname{Gal}(F, k(X))$ is doubly transitive and its order is divisible by $n(n-1)(q-1)$. To prepare the ground work for this, here we shall prove two auxilliary lemmas.

First let us note that $F$ is irreducible because it is linear in $X$, and hence $\operatorname{Gal}(F, k(X))$ is transitive. By "throwing away" the root $Y$ of $F$ we get the monic polynomial $\Omega(Y, Z)=Z^{-1}[F(Z+Y)-F(Y)]$ of degree $n-1$ in $Z$ with coefficients in $k(Y)=k(X, Y)$; for the method of "throwing away" roots and its relation to one-point stabilizers, see [2]. Since the $Y$-derivative of $F$ is a nonzero element of $k$, it follows that the $Z$-discriminant of $\Omega(Y, Z)$ is a nonzero element of $k$ and $\operatorname{Gal}(\Omega(Y, Z), k(Y))$ is isomorphic to the onepoint stabilizer of $\operatorname{Gal}(F, k(X))$. For every positive integer $u$ let $E_{u}(Y, Z)=$ $Z^{-1}\left[(Z+Y)^{u}-Y^{u}\right]$. Then clearly $\Omega(Y, Z)=E_{n}(Y, Z)+\sum_{i=1}^{h} B_{n_{i}} E_{n_{i}}(Y, Z)$. Therefore, by writing $X$ and $Y$ for $Y$ and $Z$, respectively, we get the following:

Auxilliary Lemma (2.1). With $F$ as above, let $\Omega(X, Y)$ be the monic polynomial of degree $n-1$ in $Y$ with coefficients in $k[X]$ obtained by putting

$$
\Omega(X, Y)=E_{n}(X, Y)+\sum_{i=1}^{h} B_{n_{i}} E_{n_{i}}(X, Y)
$$

where, for every positive integer $u$, by $E_{u}(X, Y)$ we are denoting the homogeneous polynomial of degree $u-1$ in $(X, Y)$ with coefficients in $k$ obtained by putting $E_{u}(X, Y)=Y^{-1}\left[(Y+X)^{u}-X^{u}\right]$. Then the $Y$-discriminant of $\Omega(X, Y)$ is a nonzero element of $k$ and hence the equation $\Omega(X, Y)=0$ gives an unramified covering of the affine line $L_{k}$. Moreover, $\operatorname{Gal}(F, k(X))$ is transitive and its one-point stabilizer is isomorphic to $\operatorname{Gal}(\Omega(X, Y), k(X))$.

Now the $k(X)$-automorphism $Y \mapsto X Y$ of $k(X)[Y]$ sends $X^{1-n} \Omega(X, Y)$ to $\Omega^{*}(X, Y)=X^{1-n} \Omega(X, X Y)=E_{n}(1, Y)+\sum_{i=1}^{h} B_{n_{i}} X^{n_{i}-n} E_{n_{i}}(1, Y)$ which is a monic polynomial of degree $n-1$ in $Y$ with coefficients in $k(X)$. Likewise the $k$-automorphism $(X, Y) \mapsto(1 / X, Y)$ of $k(X)[Y]$ sends $\Omega^{*}(X, Y)$ to $\Omega^{\prime}(X, Y)=\Omega^{*}(1 / X, Y)=E_{n}^{\prime}(Y)+\sum_{i=1}^{h} B_{n_{i}} X^{n-n_{i}} E_{n_{i}}^{\prime}(Y)$ which is a monic polynomial of degree $n-1$ in $Y$ with coefficients in $k[X]$, where for every positive integer $u$ we have put $E_{u}^{\prime}(Y)=Y^{-1}\left[(Y+1)^{u}-1\right]$. It follows that the $Y$-discriminant of $\Omega^{\prime}(X, Y)$ is a nonzero element of $k[X]$, and $\operatorname{Gal}\left(\Omega^{\prime}(X, Y), k(X)\right)$ is isomorphic to $\operatorname{Gal}(\Omega(X, Y), k(X))$. Therefore, by (2.1) we get the following:

Auxilliary Lemma (2.2). With $F$ as above, let $\Omega^{\prime}(X, Y)$ be the monic polynomial of degree $n-1$ in $Y$ with coefficients in $k[X]$ obtained by putting $\Omega^{\prime}(X, Y)=E_{n}^{\prime}(Y)+\sum_{i=1}^{h} B_{n_{i}} X^{n-n_{i}} E_{n_{i}}^{\prime}(Y)$ where, for every positive integer $u$, by $E_{u}^{\prime}(Y)$ we are denoting the monic polynomial of degree $u-1$ in $Y$ with coefficients in $k$ obtained by putting $E_{u}^{\prime}(Y)=Y^{-1}\left[(Y+1)^{u}-1\right]$. Then the $Y$-discriminant of $\Omega^{\prime}(X, Y)$ is a nonzero element of $k[X]$. Moreover, 
$\operatorname{Gal}(F, k(X))$ is transitive and its one-point stabilizer is isomorphic to $\operatorname{Gal}\left(\Omega^{\prime}(X, Y), k(X)\right)$.

\section{IRREDUCIBILITY}

As another step toward the Transitivity Lemma, let us prove the following:

Irreducibility Lemma (3.1). Let $V$ be a real discrete valuation of a field $K$ (note that then $V$ maps $K$ onto $\mathbb{Z} \cup\{\infty\})$, let $0 \leq r<d$ be integers, let $f(Y)=$ $\sum_{j=0}^{d} b_{j} Y^{d-j}$ be a polynomial of degree $d$ in $Y$ with coefficients $b_{j}$ in $K$ such that $V\left(b_{j}\right) \geq V\left(b_{0}\right)=V\left(b_{r}\right)=0<V\left(b_{d}\right)<\infty$ for $0<j<r$, and $V\left(b_{j}\right) / V\left(b_{d}\right)>(j-r) /(d-r)$ for $r<j<d$, and let

$$
s=(d-r) / \mathrm{GCD}\left(V\left(b_{d}\right), d-r\right) .
$$

Then we have the following.

(3.1.1) If $y$ is a root of $f(Y)$ in an overfield of $K$ and $W$ is an extension of $V$ to $K(y)$ with $W(y)>0$ (where we again assume $W$ to map $K(y)$ onto $\mathbb{Z} \cup\{\infty\})$, then the reduced ramification exponent e of $W$ over $V$ is divisible by $s$.

(3.1.2) If $d=d-r=s$, then $f(Y)$ is irreducible in $K[Y]$.

(3.1.3) If $f(Y)$ is irreducible in $K[Y]$ and has no multiple root in any overfield of $K$, then $\left|\mathrm{Gal}\left(f(Y) / b_{0}, K\right)\right|$ is divisible by $s$.

For a moment let the situation be as in (3.1.1). Then $W\left(b_{j}\right)=e V\left(b_{j}\right)$ for $0 \leq j \leq d$, and hence $W\left(b_{j}\right) \geq W\left(b_{0}\right)=W\left(b_{r}\right)=0<W\left(b_{d}\right)<\infty$ for $0<j<r$, and $W\left(b_{j}\right)>(j-r) W\left(b_{d}\right) /(d-r)$ for $r<j<d$. Since $W(y)>0$, we see that $W\left(b_{j} y^{d-j}\right)>W\left(b_{r} y^{d-r}\right)$ for $0 \leq j<r$; therefore, since $f(y)=0$, there must be at least two minimal $W$-value terms amongst $\left(b_{j} y^{d-j}\right)_{r \leq j \leq d}$. Now if $W(y)>W\left(b_{d}\right) /(d-r)$ then for $r \leq j<d$ we would have

$$
W\left(b_{j} y^{d-j}\right)=W\left(b_{j}\right)+(d-j) W(y)>[(j-r)+(d-j)] W\left(b_{d}\right) /(d-r)=W\left(b_{d}\right)
$$

which would contradict the existence of two minimal value terms. Likewise, if $W(y)<W\left(b_{d}\right) /(d-r)$ then for $r<j \leq d$ we would have

$$
\begin{aligned}
W\left(b_{j} y^{d-j}\right)=W\left(b_{j}\right)+(d-j) W(y) & \geq(j-r)(d-r)^{-1} W\left(b_{d}\right)+(d-j) W(y) \\
& >(j-r) W(y)+(d-j) W(y) \\
& =(d-r) W(y)=W\left(b_{r} y^{d-r}\right)
\end{aligned}
$$

which would again contradict the existence of two minimal value terms. Consequently we must have $W(y)=W\left(b_{d}\right) /(d-r)$. Therefore $e V\left(b_{d}\right) /(d-r)=$ $W(y) \in \mathbb{Z}$ and hence $e$ is divisible by $s$. This proves (3.1.1).

Next for a moment let the situation be as in (3.1.2). We can take a root $y$ of $f(y)$ in an overfield of $K$ and we can take an extension $W$ of $V$ to $K(y)$. Since $f(y)=0$, there must be at least two minimal $W$-value terms amongst $\left(b_{j} y^{d-j}\right)_{0 \leq j \leq d}$. Since $d=d-r$, we must have $W\left(b_{0}\right)=0<W\left(b_{j}\right)$ for $1 \leq j \leq$ $d$. Since $f(y)=0$ and $V\left(b_{d}\right) \neq \infty$, we must also have $y \neq 0$. Consequently $W(y)>0$ because otherwise $b_{0} y^{d}$ would be the only minimal value term. Therefore, since $s=d$, by (3.1.1) we see that the reduced ramification exponent of $W$ over $V$ is divisible by $d$ and hence it must equal $d$ and $f(Y)$ must be irreducible in $K[Y]$. This proves (3.1.2). 
Finally let the situation be as in (3.1.3). Let $y_{1}, y_{2}, \ldots, y_{d}$ be the distinct roots of $f(Y)$ in a splitting field $L$ of $f(Y)$ over $K$, and take an extension $U$ of $V$ to $L$. Since $V\left(b_{0}\right)=0 \leq V\left(b_{j}\right)$ for $1 \leq j \leq d$, we must have $U\left(b_{i}\right) \geq 0$ for $1 \leq i \leq d$. Since $y_{1} y_{2} \ldots y_{d}=(-1)^{d} b_{d} / b_{0}$ and $V\left(b_{0}\right)=0<V\left(b_{d}\right)$, we conclude that $U\left(y_{i}\right)>0$ for some $i$. Let $y=y_{i}$ and let $W$ be the extension of $V$ to $K(y)$ such that $U$ is an extension of $W$ to $L$. Now $W(y)>0$ and hence by (3.1.1) we see that the reduced ramification exponent of $W$ over $V$ is divisible by $s$. Therefore $\left|\mathrm{Gal}\left(f(Y) / b_{0}, K\right)\right|$ is divisible by $s$. This proves (3.1.3).

\section{TRANSITIVIty}

Finally let us state and prove the:

Transitivity Lemma (4.1). Let $F=F(Y)=Y^{n}+B_{n_{1}} Y^{n_{1}}+B_{n_{2}} Y^{n_{2}}+\cdots+B_{n_{h}} Y^{n_{h}}+$ $X$ where $h$ and $n>n_{1}>n_{2}>\cdots>n_{h}=1$ are positive integers, and $0 \neq$ $B_{n_{i}} \in k$ for $1 \leq i \leq h$. Assume that $n$ is divisible by $p$ and let $m$ and $q$ be the unique positive integers with $n=m q$ such that $m$ is nondivisible by $p$ and $q$ is a power of $p$. For $1 \leq i \leq h-1$ assume that $n_{i}$ is divisible by $p$ and let $m_{i}$ and $q_{i}$ be the unique positive integers with $n_{i}=m_{i} q_{i}$ such that $m_{i}$ is nondivisible by $p$ and $q_{i}$ is a power of $p$. [Note that the $Y$-derivative of $F$ equals the nonzero element $B_{n_{h}}$ of $k$ and hence the Galois group $\operatorname{Gal}(F, k(X))$ makes sense and the equation $F=0$ gives an unramified covering of the affine line $\left.L_{k}.\right]$ Now considering the conditions

$(*)$

$\operatorname{GCD}(n-1, q-1)=1$ and $\left(q_{i}-1\right)(n-1)>(q-1)\left(n_{i}-1\right)$ for $1 \leq i \leq h-1$,

and

$$
\left(n-n_{i}\right)(q-1)>(n-1)\left(q-q_{i}\right) \text { for } 1 \leq i \leq h-1,
$$

we have that: $(*) \Rightarrow \operatorname{Gal}(F, k(X))$ is doubly transitive, and $(*)+(* *) \Rightarrow$ $|\mathrm{Gal}(F, k(X))|$ is divisible by $n(n-1)(q-1)$.

To prove (4.1), in view of (2.2), it suffices to show that in the situation of $(2.2), \quad(*) \Rightarrow \Omega^{\prime}(X, Y)$ is irreducible in $k(X)[Y]$, and $(*)+(* *) \Rightarrow$ $\left|\mathrm{Gal}\left(\Omega^{\prime}(X, Y), k(X)\right)\right|$ is divisible by $q-1$. So let the situation be as in (2.2) and assume $(*)$, let $K=k(X)$ and $d=n-1$, and let $V$ be the order of zero at $X=0$, i.e., $V\left(X^{t} P(X) / Q(X)\right)=t$ for all integers $t$ and all $P(X)$ and $Q(X)$ in $k[X]$ with $P(0) \neq 0 \neq Q(0)$. Note that now $V\left(E_{n}^{\prime}(X)\right)=q-1$, $V\left(E_{n_{i}}^{\prime}(X)\right)=q_{i}-1$ for $1 \leq i \leq h-1$, and $E_{n_{h}}^{\prime}(X)=1$.

For a moment let $r=0$ and $f(Y)=\Omega^{\prime}(Y, X)=\sum_{j=0}^{d} b_{j} Y^{d-j}$ with $b_{j} \in K$. Then $b_{0}=B_{n_{h}} E_{n_{h}}^{\prime}(X)=$ the nonzero element $B_{n_{h}}$ of $k$. Also $b_{d}=E_{n}^{\prime}(X)$ and hence $V\left(b_{d}\right)=q-1$. Moreover, for $1 \leq i<h$ we have $0<n_{i}-1<d$ and $b_{n_{i}-1}=B_{n_{i}} E_{n_{i}}^{\prime}(X)$ and hence $V\left(b_{n_{i}-1}\right)=q_{i}-1$ and therefore $V\left(b_{n_{i}-1}\right) / V\left(b_{d}\right)>$ $\left(n_{i}-1\right) / d$. Clearly $b_{j}=0$ for all $j \in\{1,2, \ldots, d\} \backslash\left\{n_{1}, n_{2}, \ldots, n_{h}\right\}$, and hence $V\left(b_{j}\right) / V\left(b_{d}\right)>j / d$ for $0<j<d$. Since GCD $(n-1, q-1)=1$, we also get $\operatorname{GCD}\left(V\left(b_{d}\right), d\right)=1$, and hence upon letting $s=(d-r) / \operatorname{GCD}\left(V\left(b_{d}\right), d-r\right)$ we have $d=d-r=s$. Consequently by (3.1.2) we conclude that $\Omega^{\prime}(Y, X)$ is irreducible in $k(X)[Y]$. Therefore $\Omega^{\prime}(X, Y)$ is irreducible in $k(Y)[X]$, and hence by Gauss's Lemma we see that $\Omega^{\prime}(X, Y)$ is irreducible in $k(X)[Y]$. 
Now assume $(* *)$ and let $r=n-q$ and $f(Y)=\Omega^{\prime}(X, Y)=\sum_{j=0}^{d} b_{j} Y^{d-j}$ with $b_{j} \in K$. Then $V\left(b_{j}\right) \geq V\left(b_{0}\right)=V\left(b_{r}\right)=0<V\left(b_{d}\right)=n-1=$ $d$ for $0<j<r$, and $V\left(b_{j}\right) / V\left(b_{d}\right)>(j-r) /(d-r)$ for $r<j<d$. Also $(d-r) / \operatorname{GCD}\left(V\left(b_{d}\right), d-r\right)=q-1$. Therefore by (3.1.3) we see that $\left|\operatorname{Gal}\left(\Omega^{\prime}(X, Y), k(X)\right)\right|$ is divisible by $q-1$.

\section{LINEARIZATION}

Let us now prove the:

Linearization Lemma (5.1). If $p=2$ and $T$ is any element in an overfield of $k(X)$ [for instance, $T$ could be transcendental over $k(X)$ ], then there exist elements $A_{0}, A_{1}, \ldots, A_{12}$ in $k[X, T]$ with $A_{0} \neq 0$ and $A_{12}=1$ such that $\sum_{i=0}^{12} A_{i} Y^{2^{i}}=H F^{*}$ for some $H \in k[X, T][Y]$ where $F^{*}=Y \bar{F}_{23,20,1,1}+T$.

The proof of (5.1) is simply obtained by adding obvious terms involving $T$ in the RHS of various equations occurring in the proof of (1.5) of [4] given in Section 5 of [4]. In greater detail: To prove (5.1) assume that $p=2$. Now

$$
F^{*}=Y^{24}+X Y^{4}+Y+T
$$

and by adding $F^{*}+Y^{24}$ to both sides of this we get

$$
Y^{24}=X Y^{4}+Y+T+F^{*} .
$$

Let $P \equiv Q$ mean $P-Q=H F^{*}$ for some $H \in k[X, T][Y]$. Then multiplying $\left(J_{24}^{\prime}\right)$ by $Y^{i-24}$ for $i=24,26,32,36$ we get:

$$
\begin{gathered}
Y^{24} \equiv X Y^{4}+Y+[T], \\
Y^{26} \equiv X Y^{6}+Y^{3}+\left[T Y^{2}\right], \\
Y^{32} \equiv X Y^{12}+Y^{9}+\left[T Y^{8}\right], \\
Y^{36} \equiv X Y^{16}+Y^{13}+\left[T Y^{12}\right] .
\end{gathered}
$$

Squaring $\left(J_{32}\right)$ we get

$$
Y^{64} \equiv X^{2} Y^{24}+Y^{18}+\left[T^{2} Y^{16}\right]
$$

and using $\left(J_{24}\right)$ we obtain

$$
Y^{64} \equiv Y^{18}+X^{3} Y^{4}+X^{2} Y+\left[T^{2} Y^{16}+X^{2} T\right] .
$$

Likewise, by squaring $\left(J_{64}\right)$ and then using $\left(J_{36}\right)$ we obtain

$\left(J_{128}\right) \quad Y^{128} \equiv X Y^{16}+Y^{13}+X^{6} Y^{8}+X^{4} Y^{2}+\left[T^{4} Y^{32}+T Y^{12}+X^{4} T^{2}\right]$.

Again, by squaring $\left(J_{128}\right)$ and then using $\left(J_{24}\right),\left(J_{26}\right)$, and $\left(J_{32}\right)$ we obtain

$$
\begin{aligned}
Y^{256} \equiv & X^{12} Y^{16}+X^{3} Y^{12}+X^{2} Y^{9}+X Y^{6}+X^{8} Y^{4}+Y^{3} \\
& +\left[T^{8} Y^{64}+X^{2} T Y^{8}+X T^{2} Y^{4}+T Y^{2}+T^{2} Y+X^{8} T^{4}+T^{3}\right] .
\end{aligned}
$$

Similarly, by squaring $\left(J_{256}\right)$ and then using $\left(J_{24}\right)$ and $\left(J_{32}\right)$ we obtain $\left(J_{512}\right)$

$$
\begin{gathered}
Y^{512} \equiv X^{4} Y^{18}+\left(X^{2}+X^{25}\right) Y^{12}+X^{24} Y^{9}+X^{16} Y^{8}+Y^{6}+X^{7} Y^{4}+X^{6} Y \\
+\left[T^{16} Y^{128}+X^{4} T^{2} Y^{16}+\left(X^{2} T^{4}+X^{24} T\right) Y^{8}\right. \\
\left.+T^{2} Y^{4}+T^{4} Y^{2}+\left(X^{16} T^{8}+T^{6}+X^{6} T\right)\right] .
\end{gathered}
$$


Likewise, by squaring $\left(J_{512}\right)$ and then using $\left(J_{24}\right)$ and $\left(J_{36}\right)$ we obtain

$$
\begin{aligned}
Y^{1024} \equiv & X^{48} Y^{18}+\left(X^{9}+X^{32}\right) Y^{16}+X^{8} Y^{13}+Y^{12} \\
& +X^{14} Y^{8}+\left(X^{5}+X^{51}\right) Y^{4}+X^{12} Y^{2}+\left(X^{4}+X^{50}\right) Y \\
\left(J_{1024}\right) \quad & {\left[T^{32} Y^{256}+X^{8} T^{2} Y^{32}+\left(X^{4} T^{8}+X^{48} T^{2}\right) Y^{16}+X^{8} T Y^{12}\right.} \\
& +\left(T^{4}+X^{18} T+X^{64} T\right) Y^{8}+T^{8} Y^{4} \\
& \left.\quad+\left(X^{32} T^{16}+T^{12}+X^{12} T^{2}+X^{50} T+X^{4} T\right)\right] .
\end{aligned}
$$

Finally, by squaring $\left(J_{1024}\right)$ and then using $\left(J_{24}\right),\left(J_{26}\right),\left(J_{32}\right)$, and $\left(J_{36}\right)$ we obtain

$$
\begin{aligned}
\left(J_{2048}\right) & \\
Y^{2048} \equiv & \left(X^{28}+X^{97}\right) Y^{16}+X^{96} Y^{13}+\left(X^{19}+X^{65}\right) Y^{12} \\
& +\left(X^{18}+X^{64}\right) Y^{9}+\left(X^{10}+X^{102}\right) Y^{8}+X^{17} Y^{6} \\
& +\left(X+X^{24}\right) Y^{4}+X^{16} Y^{3}+\left(X^{8}+X^{100}\right) Y^{2}+Y \\
& +\left[T^{64} Y^{512}+X^{16} T^{4} Y^{64}+\left(X^{8} T^{16}+X^{96} T^{4}\right) Y^{32}\right. \\
& +\left(T^{8}+X^{36} T^{2}+X^{128} T^{2}\right) Y^{16}+X^{96} T Y^{12} \\
& +\left(T^{16}+X^{18} T+X^{64} T\right) Y^{8}+X^{17} T^{2} Y^{4}+X^{16} T Y^{2}+X^{16} T^{2} Y \\
& \left.\quad+\left(X^{64} T^{32}+T^{24}+X^{24} T^{4}+X^{16} T^{3}+X^{100} T^{2}+X^{8} T^{2}+T\right)\right] .
\end{aligned}
$$

Since the above formulas $\left(J_{24}\right),\left(J_{26}\right), \ldots,\left(J_{2048}\right)$ are obtained by adding $T$-terms in the RHS of the corresponding formulas $\left(I_{24}\right),\left(I_{26}\right), \ldots,\left(I_{2048}\right)$ of Section 5 of [4], by modifying the last formula of that section to compensate for the $T$-terms we get

$$
\begin{aligned}
& Y^{2048}+T^{64} Y^{512}+X^{16} Y^{256}+X^{96} Y^{128}+\left(X^{8} T^{16}+X^{64}\right) Y^{32} \\
& \quad+\left(T^{16}+X^{10}\right) Y^{8}+X Y^{4}+X^{8} Y^{2}+Y+\left(X^{64} T^{32}+T^{24}+X^{8} T^{2}+T\right) \equiv 0 .
\end{aligned}
$$

Alternatively the above equation can be proved directly by using $\left(J_{2048}\right),\left(J_{512}\right)$, $\left(J_{256}\right),\left(J_{128}\right)$, and $\left(J_{32}\right)$. By multiplying the above equation by its constant term $X^{64} T^{32}+T^{24}+X^{8} T^{2}+T$ and then adding the resulting equation to the square of the above equation we get

$$
\begin{aligned}
Y^{4096} & +\left(X^{64} T^{32}+T^{24}+X^{8} T^{2}+T\right) Y^{2048}+T^{128} Y^{1024} \\
& +\left(X^{64} T^{96}+T^{88}+X^{8} T^{66}+T^{65}+X^{32}\right) Y^{512} \\
& +\left(X^{80} T^{32}+X^{16} T^{24}+X^{24} T^{2}+X^{16} T+X^{192}\right) Y^{256} \\
& +\left(X^{160} T^{32}+X^{96} T^{24}+X^{104} T^{2}+X^{96} T\right) Y^{128}+\left(X^{16} T^{32}+X^{128}\right) Y^{64} \\
& +\left(X^{72} T^{48}+X^{8} T^{40}+X^{128} T^{32}+X^{64} T^{24}\right. \\
& \left.+X^{16} T^{18}+X^{8} T^{17}+X^{72} T^{2}+X^{64} T\right) Y^{32}+\left(T^{32}+X^{20}\right) Y^{16} \\
& +\left(X^{64} T^{48}+T^{40}+X^{74} T^{32}+X^{10} T^{24}\right. \\
& \left.+X^{8} T^{18}+T^{17}+X^{18} T^{2}+X^{10} T+X^{2}\right) Y^{8} \\
& +\left(X^{65} T^{32}+X T^{24}+X^{9} T^{2}+X T+X^{16}\right) Y^{4} \\
& +\left(X^{72} T^{32}+X^{8} T^{24}+X^{16} T^{2}+X^{8} T+1\right) Y^{2} \\
& +\left(X^{64} T^{32}+T^{24}+X^{8} T^{2}+T\right) Y \equiv 0
\end{aligned}
$$

and this proves $(5.1)$. 


\section{MATHIEU GROUP}

To prove (1.1) assume that $p=2$, let $\alpha$ be any element of $k$, and let $G=\operatorname{Gal}\left(Y^{24}+\alpha Y^{4}+Y+X, k(X)\right)$. Then by (4.1) we see that $G$ is a doubly transitive permutation group of degree 24 whose order is divisible by 7 , and hence by CTT and Special CDT on pp. 86 to 89 of [2], we must have $G=M_{24}$ or $A_{24}$ or $S_{24}$. As said in the Introduction, by taking $(\alpha, X)$ for $(X, T)$ in $(5.1)$ we see that $|G|$ divides $|\mathrm{GL}(12,2)|$. Finally, as a factorization of $|\mathrm{GL}(12,2)|$ into powers of prime numbers we have

$$
\begin{aligned}
|\mathrm{GL}(12,2)| & =\prod_{i=0}^{11}\left(2^{12}-2^{i}\right) \\
& =2^{66} \times 3^{8} \times 5^{3} \times 7^{4} \times 11 \times 13 \times 17 \times 23 \times 31^{2} \times 73 \times 89 \times 127,
\end{aligned}
$$

but $\left|A_{24}\right|$ and $\left|S_{24}\right|$ are obviously divisible by $11^{2}$ and hence we must have $G=M_{24}$.

\section{REFERENCES}

1. S. S. Abhyankar, Coverings of algebraic curves, Amer. J. Math. 79 (1957), 825-856.

2. __ Galois theory on the line in nonzero characteristic, Bull. Amer. Math. Soc. (N.S.) 27 (1992), 68-133.

3. Bombay International Colloquium on Geometry and Analysis held at the Tata Institute of Fundamental Research (to appear).

4. $ـ$ Mathieu group coverings in characteristic two, C. R. Acad. Sci. Paris Sér. I Math. 316 (1993), 267-271.

5. S. S. Abhyankar and I. Yie, Small degree coverings of the affine line in characteristic two, Discrete Math. (to appear).

6. J.-P. Serre, e-mail to Abhyankar dated October 1991.

Department of Mathematics, Purdue University, West Lafayette, Indiana 47907

E-mail address: ramecs.purdue.edu

E-mail address : yie@math.purdue.edu 